\title{
Contributiones ad Floram Asiae Orientalis
}

(Continued from vol. XXXII. p. 259.)

by

Geniti Koizumi Rigakuhakushi

Lysimachia (Lubinia) rubida Kordz. n. sp.

A Lysimachia Mauritiana LAM. cui habitu haud dissimili, floribus majoribus pedicellis brevioribus, calycis lobis lanceolatis acutis maturitate fructibus aequilongis, fructu majore apice attenuato brevius pedicellato recedit.

Glabra 1-6 dm. alta. Caulis erectus simplex vel e basi et superne ramosus saepe nigro-punctatus, ramis erectis. Folia tenuiter carnosula alterna subtus in specimina sicca albicantia, utrinque dense nigropunctata, spathulata vel lineari-oblanceolata obtusa, margine integerrima et leviter revoluta, versus basim longe vel breviter attenuata, basi dilatata et plus minus decurrentia, 3-61/2 cm. longa, 10-13 $\mathrm{mm}$. lata, superiora oblongo-spathulata vel lineari-oblonga interdum ovatooblonga sessilia. Racemus ad $14 \mathrm{~cm}$. longus; floribus 3-circ. 30, albis vel rubris circ. $18 \mathrm{~mm}$. longis sessilibus vel brevissime pedicellatis, pedicellis mox elongatis. Calyx campanulatus circ. ad $9 \mathrm{~mm}$. altus profunde 5-partitus, laciniis lanceolatis obtusis extus carinato-rotundatis intus nigro-punctatis. Corolla tubo $4-5 \mathrm{~mm}$. longo, lobis 5 obovatooblongis acutiusculis $12-13 \mathrm{~mm}$. longis mox leviter revolutis apice pluripunctatis. Stamina 5 circ. $9 \mathrm{~mm}$. longa, filamentis subulatis ad corollae lobum longe adnatis, antheris ovatis obtusis connectivo nigrescente. Ovarium cylindrico-ovoideum circ. $3 \mathrm{~mm}$. altum extus nigro-striatum, stylis subulatis teretibus circ. $11 \mathrm{~mm}$. longis, stigma capitata. Capsula ovoidea circ. $5 \mathrm{~mm}$. lata $8-9 \mathrm{~mm}$. alta apice breviter attenuata stylis rectis circ. $10 \mathrm{~mm}$. longis persistentibus coronata.

Nom. Jap. Oh-hamabossu (nov.)

Distr. Bonin: insl. (hichishima, ad rupibus Hatsuneyama (lg. S. Nishimura! no. 158.) 
Polygonatum macranthum (MAXim.)

Convallaria multiflora Thuns. Fl. Jap. 142. (non Linv.)

Polygonatum canaliculatum MiQ. Prol. Fl. Jap. $148 ;-F_{R}$. et SAv. Enum. P1. Jap. II. 54. (non PuRsH.)

P. latifolium var. commutatum BAKER Jour. Linn. Soc. XIV. 555, (quoad pl. Jap.)

$P$. giganteum var. macranthum MAXIM. in Mel. Biol. XI. 852. (1883).

? P. canaliculatum, $\alpha$. sublanceolatum MiQ. Prol. Fl. Jap. 148. (1866).

? P. falcatum f. robustum Mak. in IrNuma Somoku Dsusetsu. VI. t. 6.

? P. Thunbergii Morr. et Decne. Obs. 5, in Ann. d. Sc. Nat. Nov. 2 sér. II. (1834), 135.

Haec planta Polygonatis commutatis Dietr. et giganteis Dietr. habitu approximat, ab illo differt floribus majoribus (32-34 $\mathrm{mm}$. $1 \mathrm{~g}$.) viridibus, pedunculis 2-3-floris, caule non anguloso; ab hoc floribus majoribus, filamentis plerumque glabris partibus liberis quam antheræ longioribus, pedunculis 2-3-floris, foliis lanceolato-oblongis vel oblongolanceolatis acuminatisque distincta.

Glabrum caule tereti robusto arcuato 2-4-ped. alto. Folia tenue membranacea supra viridia subtus glaucescentia, lanceolato-oblonga vel oblongo-lanceolata apice attenuata 5-10-nervia brevissime petiolata, 10-21 cm. longa, 3-8 cm. lata. Pedunculi 2-3-flori cernui floribus 30-35 $\mathrm{mm}$. longis viridibus. Perianthium tubuloso-campanulatum leviter 6-lobum lobis exterioribus ovatis obtusissimis, interioribus ovatis rotundatis, omnibus apice puberulis. Filamenti pars liber glaber quam anthera longior.

Nom. Jap. Ohba-wösei.

Drstr. Japonia : Nippon, Shikoku, Kiushiu.

Polygonatum odoratum (Mrle.) Druce in Ann. Nat. Hist. Soc. Scot. (1906) p. 226.

Convallaria odorata MrLL. Gard. Dict. ed. 8, no. 3, (1768).

Polygonatum officinale ALL. Fl. Pedem I. (1785) 131.

P. japonicum Morr. et DeCne. in Ann. Sc. Nat. 2 ser. II. (1834) p. 311.

Nom. Jap. Amadokolo.

DisTR. regionibus temperatis Eurasiae.

var. Maximowiczii (Fr. SchMd )

Polygonatum Maximowiczii Fr. Schmd. Reis. no. 449. (1868).

P. officinale var. Maximowiczii Maxım. Mel. Biol. XI. (1883) 847.

Noм. Jap. Oh-amadokolo.

Distr. Sachalin, Yezo, Nippon, Amur, China borealis. 
Polygonatum trichosanthum KoIdz. n. sp.

Haec species affinis Polygonatis latifoliis Desf. sed ab eo differt caule non anguloso glabro, foliis ovatis vel elliptico-ovatis, pedicello glabro, perigonio filamentisque pilosis; a Polygonatis multifloris AlL. foliis subtus ad nervos puberulis, floribus latioribus intus glabris extus pilosisque diversa.

Caulis ultra $6 \mathrm{dm}$. altus glaber teres. Folia chartaceo-membranacea subtus ad venas scabra et leviter glaucina ovata obtusa $71 / 2-13 \frac{1}{2} \mathrm{~cm}$. longa, 21/2-51/2 cm. lata, 7-10-costata. Pedunculi glabri 3-4-flori, floribus circ. $18 \mathrm{~mm}$. longis. Perianthium extus hirtum apice leviter 6-lobum, lobis ovatis obtusis apice puberulis intus pilosis. Filamenti pars liber laxe hirta quam anthera longior.

Noм. JAP. Maluba-wōsei.

HaB. in Hort. Bot. Koishikawensi, Tokyo, Japan.

Polygonatum tenuiflorum KorDz. n. sp.

A $P$. commutato differt foliis lanceolato-oblongis subtus ad nervos scabris floribus glabris angustioribus.

Caulis glaber $5-5 \frac{1}{2} \mathrm{dm}$. altus teres basi leviter subangulatus. Folia tenue membranacea supra viridia subtus glaucina et ad nervos scabra, ovato-oblonga obtusa vel lanceolato-oblonga acuta, 3-5-costata, basi subito angustata, 7-91/2 cm. longa, 17-30 mm. lata. Pedunculi biflori cernui glabri, floribus 15-17 $\mathrm{mm}$. longis 3-4 $\mathrm{mm}$. latis. Perianthium leviter 6-lobum, laciniis ovatis obtusis apice puberulis. Filamenta glabra quam anthera longiora.

Nom. JAP. Hosoba-Konalukoyuri.

Distr. Nippon: Prov. Suwō, Yoshikigōri, Ohuchimura, (lg. T. NikaI!)

\section{Disporum lutescens (Maxim.)}

D. smilacinum var. lutescens Maxim. in Mel. Biol. XI. (1883) p. 859.

Species insignis foliis supremis acuminatis, perigonii phyllis lanceolatis acuminatis.

Nom. JaP. Kibana-chigoyuri.

Distr. Kiushiu: Kumamoto, Iwadomura (Prov. Hiuga, Nishiusukigōri). Korea.

Pennisetum (Gymnothrix) sordidum Kordz. n. sp.

Haec species $P$. compressis $R$. BR. affinis sed spica densiflora spiculis fere sessilibus culmo laeve differt; etiam $P$. purpurascenti var. viridescenti Makino comparanda sed foliis convolutis floribus duplo minoribus fere sessilibus, gluma II uninervia, lemma acuta 5-nervia, palea acuminata, stylis liberis, culmo laeve glabroque recedit. 
Radix perennis rhizomate lignoso. Culmi dense caespitosi, erecti, simplices vel e basi pauci-ramosi, laeves et glabri, 3-4 dm. alti, circ. 7-8-nodi, nodis inferioribus 3-4 aproximatis. Vaginae internodio multo longiores, compressae, glaberrimae, ligula brevissima fere $1 / 2 \mathrm{~mm}$. longa vaginarum inferiorum membranacea, superiorum in fimbrias densissimis soluta. Lamina rigide coriacea glabra tubuloso-convoluta apice acuminata plerumque culmo superantia. Thyrsus viridis densiflorus 5-8 cm. longus erectus vel apice subnutans, rhachibus sulcato-angulatis dense pilosis; pedicellis $1 / 2-3 / 4 \mathrm{~mm}$. longis dense pilosis. Spiculae acutissimae circ. $51 / 2 \mathrm{~mm}$. langae, involucri setis sordidis vel pallide fulvis 2-20 mm. longis minute hispidis. Grumae inferiores rotundatae minutae, superiores oblongae $1,8 \mathrm{~mm}$. longae uninerviae obtusissimae. Lemma ovata acuta $4 \mathrm{~mm}$. longa 5-nervia. Palea lanceolato-ovata acuminata $51 \frac{1}{2} \mathrm{~mm}$. langa 5-nervia. Lodicula linearia $5 \mathrm{~mm}$. longa. Stamina 3. Ovarium ellipsoideum stylis 2 basi vix connatis, stigmatibus linearibus dense plumosis.

Nom. Jap. Shima-chikalashiba.

Type locality : Bonin, (leg. S. Nishimura!)

Range: Kiushiu : Prov. Ohsumi, insl. Amami-ohshima (lg. T. Uсhiу AMa! Dec. 1900.)

Platanthera (Bifolia) boninensis Kordz. n. sp.

Proxima PI. Okuboi MAK. quæ floribus majoribus, petalis ovatolinearibus vel oblongo-linearibus trinerviis, calcare $2 \frac{1}{2}-3 \mathrm{~cm}$. longo differt.

Terrestris glaberrima erecta $20-50 \mathrm{~cm}$. alta ; caule tereti robusto basi cataphyllis tribus $1-5 \mathrm{~cm}$. longis vaginato. Folia 5-10; foliis inferioribus 2 vel 3 majoribus fere aequalibus vel inæqualibus erectopatentibus, ovalibus vel ovatis raro late ellipticis interdum oblongis 7-13 cm. longis $4-51 / 2 \mathrm{~cm}$. latis, a pice rotundatis vel obtusissimis, basi amplectentibus et decurrentibus, integerrimis, membranceis, utrinque 9-16-costatis ; foliis superioribus multo minoribus adpressis vel erectopatentibus lanceolatis, obtuse attenuatis semiamplexicaulibus, 1-6 cm. longis, infimis ad $81 / 2 \mathrm{~cm}$. longis et $3 \mathrm{~cm}$. latis. Racemi erecti $5-12 \mathrm{~cm}$. longi multiflori, bracteis erecto-patentibus foliaceis lanceolatis vel ovato-lanceolatis, obutuse acuminatis ovario longioribus membranaceis 3-plurinervosis usque $2 \mathrm{~cm}$. longis $7 \mathrm{~mm}$. latis ; floribus viridescentibus? Sepala membranacea terminalibus erectis ovalibus apice rotundatis trinervis $4 \mathrm{~mm}$. longis; lateralibus patentireflexis oblongis apice rotundatis trinervis $5 \mathrm{~mm}$. longis. Petala erecta oblique ovata obtusa $3 \frac{1}{2} \mathrm{~mm}$. longa sub-5-nervia. Labellum lanceplato-lineare apice 
obtusissimum vel integerrimum $5 \mathrm{~mm}$. longum ; calcare anguste elongato

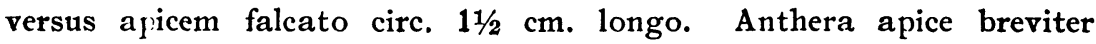
emarginata, rostello valde humili obtusissimo, ovario angusta to sessili arcuato versus apicem sensim attenuato circ. $11 \mathrm{~mm}$. longo. Capsula cylindracea $13 \mathrm{~mm}$. longa $31 / 2 \mathrm{~mm}$. lata.

Nom. Jap. Shima-tsulesagi.

Distr. Bonin : insl. Chichishima (lg. B. Kawate! S. NishiMURA! J. Toyoshima!)

\section{Salix Hisauchiana $q$ n. sp.}

vel Hybr. (=S. japonica $\times$ multinervis)

Planta insignis foliis obovato-oblongis vel ovato-oblongis glabris obtusis obscuriter denticulatis basi subcordatis subtus glaucescentibus.

Frutex breviter ramosus, ramuli novelli glabri, biennes castanci, vetustiores sordide cinerascentes. Folia alterna, juvenilia glabra supra viridia subtus vix glaucina obovato-oblonga vel obovato-elliptica raro ovato-oblonga vel lanceolato-oblonga apice obtusa raro acuta vel obtusissima, basi rotundato-cordata, margine minutissime denticulata ; petiolis brevissimis circ. 1-2 mm. longis, stipulis obsoletis. Amenta coetanea brevipedunculata basi foliis parvis oblongis apice rotundatis 2-4. suffulta, cylindrica densiflora 3-2 cm. longa, rhachi sericeo-villosa, ovaria brevissime stipitata ovato-oblonga laxe sericeo-puberula, stylis brevissimis apice bifidis, stigmatibus brevibus cmarginatis; glandula una ventralis late ovata apice truncata quam stipitus longiora; bracteolis oblongo-linearibus glabris apice rotundatis rubro vel purpureocoloratis.

NoM. JaP. Fuji-yanagi.

Distr. Nippon: Mt. Fujisan (lg. Kiyotaka Hisauchi! V. 19, 1918.)

Cirsium (Onotrophe) heiianum KoIDz. n. sp.

Quoad habitum $C$. nipponico var. amplexifolio valde affine, sed capitula eis $C$. bitchuensis simillima.

Species insignis involucri squamis purpureis extimis intimis multo brevioribus reflexis, foliis linearibus vel oblanceolato-linearibus integris margine creberrime spinulosis.

Caulis elatus circ. $7 \mathrm{dm}$. altus striato-sulcatus parce villosus superne ramosus crebre foliatus, ramis erectis gracilibus. Folia membranacea supra leviter papillosa subtus puberula; inferiora pandulaeformia vel oblanceolato-oblonga acutissima basi amplexicaulia suberoso-serrata spinoso-ciliataque, dentibus spinoso-acuminatis, lamina $16-23 \mathrm{~cm}$. longa $31 \frac{1}{2}-8 \mathrm{~cm}$. lata; folia superiora linearia raro lanceolato-linearia acutissima 
sessilia 6-12 $\mathrm{cm}$. longa 7-18 $\mathrm{mm}$. lata, margine spinoso-ciliata. Capitula ad apices ramorum 1-3, erecta, ovata raro campanulata basi rotundata $21 / 2-3 \mathrm{~cm}$. longa, basi 5-6 mm. lata, pedicellis brevissimis puberulis ; involucri squamis extimis ovatis acutissimis vel spinoso-acutis $3 \mathrm{~mm}$. longis, interioribus linearibus $14 \mathrm{~mm}$. longis superne purpureis fimbriato-marginatis 1-3 mm. latis. Flores purpurei corollae tubo filiforme $7 \mathrm{~mm}$. longo, limbo basi subito constricto $8 \mathrm{~mm}$. longo; stylo superne nodoso-incrassato ad nodum penicillato, filamentis dense papillosis. Achaenium album $3 \frac{1}{2} \mathrm{~mm}$. longum, pappo $10-13 \mathrm{~mm}$. longo candido.

Nom. JaP. Tōno-azami.

Distr. Nippon: Prov. Rikuchiu, Tōno, Monomiyama.

Cirsium (Onotrophe) chikushiense Koldz. n. sp.

Cirsio nikkoensi NAKaI remote affine, differt foliis minus dissectis cauleque non lanuginosis, capitulis interdum non aggregatis basi foliis involucrantibus suffultis, involucri squamis non erectisque.

Caulis elatus ramosus ultra tripedalis sulcatus araneosus versus apicem villoso-tomentosus, ramis erectis. Folia coriacea rigida lanceolata pinnatifida ad $25 \mathrm{~cm}$. longa et $9 \mathrm{~cm}$. lata, acuminata sessilia utrinque laxius araneosa, laciniis subdigitatim grosse dentatis, dentibus apice spinis validis longis terminatis. Capitula ovata ad apices caulis ramorumque 2-3 approximata interdum remota et breviter pedunculata, $3-3 \frac{1}{2} \mathrm{~cm}$. lata, basi foliis brevibus involucrantibus suffulta. Involucrum plus mimus araneosum, squamis exterioribus lanceolatis erecto-patentibus vel superne leviter recurvatis apice aristatis, interioribus linearibus acutis. Flores purpurei corollae tubo limbo ampliato vix breviore, staminorum filamentis dense papillosis, styli nodo glabro, pappo candido.

Nom. Jap. Noma-azami.

Distr. Kiushiu: Prov. Satsuma, Nomagatake.

Cirsium (Eriolepis) Toyoshimae Koldz. n. sp.

Quoad habitum Cirsio effuso similis, sed capitulum eis C. aomorensis simillimum.

Caule elato superne ramoso parce arachnoideo striato-sulcato, ramis monocephalis superne albovillosis; foliis membranaceis utrinque parce puberulis lanceolatis, apice obtusais sed spinoso-mucronatis basi late cuneatis breviter petiolatis utrinque grosse 2-3-dentatis et remote spinosis; foliis ramulorum integris vel pauci-spinosodentatis ; capitulis globosis basi $14 \mathrm{~mm}$. latis excavatis $2 \frac{1}{2} \mathrm{~cm}$. longis, sub anthesi erect is ; involucri squamis intimis linearibus acutis $14 \mathrm{~mm}$. longis erectis, extimis 
conformibus circ. $6 \mathrm{~mm}$. longis interioribusque apice leviter recurvis. Flores purpurei tubofi liforme $8 \mathrm{~mm}$. longo limbum basi attenuatum aequilongo; stylis versus apicem penicillato-nodosis; filamentis dense puberulis ; pappo candido.

Nom. Jap. Toshima-azami.

Distr. Bonin (lg. J. Toyoshima! no. 81.)

Cirsium (Eriolepis) Ogatae KoIDz. n. sp.

Quoad habitum C. pendulo affine sed floribus valde diversis capitulo eis C. aomorensis affine.

Caulis elatus ultra tripedalis valide angulato-striatus arachnoideovillosus vel glabrescens superne ramosus, ramis albo-villosis apice monocephalis vel spicato-tricephalis. Folia chartaceo-membranacea utrinque parce pilosa vel subtus vix arachnoidea, ambitu elliptica vel oblonga utrinque 4-5-pinnatifida, apice acuminata basi brevissime petiolata, lobis lanceolatis acuminatis grosse pauci-pluridentatis, dentibus apice spinis ad $10 \mathrm{~mm}$. longis terminatis; foliis inferioribus 40 $\mathrm{cm}$. longis $25 \mathrm{~cm}$. latis, lobis 14 . cm. longis, supremis $13 \mathrm{~cm}$. longis $5 \frac{1}{2}$ cm. latis, lobis ambitu lanceolato-ovatis $3 \frac{1}{2} \mathrm{~cm}$. longis $1 \frac{11 / 2}{\mathrm{~cm}}$. latis. Capitula globosa basi $13 \mathrm{~mm}$. lata excavata $3 \frac{1}{2} \mathrm{~cm}$. alta, basi plerumque nuda, interdum foliis lanceolato-linearibus $4 \frac{1}{2} \mathrm{~cm}$. longis versus basim pluridentatis suffulta; involucri squamis araneosis erectis lineari-lanceolatis acuminatis, extimis $10 \mathrm{~mm}$. longis, intimis $20 \mathrm{~mm}$. longis ; flosculis purpureis corollae tubo $9 \mathrm{~mm}$. longo limbum aequante ; filamentis dense puberulis, pappo sordido.

Nom. Jar. Ogata-azami.

Distr. Kiushiu ; Prov. Hiuga, Nishiusukigōri, Iwadomura, Mitate, (lg. Matsuzö Ogata! Aug. 20, 1913.)

\section{Putranjixa Matsumurae Kordz. n. sp.}

P. Roxburghii (non WaI.L.) Matsum. in Bot. Mag. Tokyo, XII. (1898) 61 ;-Hayat. Rev. Euphorb. Jap. in Jour. Coll. Sci. Imp. Univ. Tokyo, vol. XX. art. 3, p.p. 24-26, (1904).

A. P. Roxburghii W ALL. differt sepalis florum $\hat{0}$ 4-6, staminibus 2 ; sepalis florum $\&$ 3-4, stigmatibus bilobis non flabellatis.

A. P. ceylanica differt sepalis $\uparrow$ 4-6, \& 3-4, ovario triloculare, foliis basi inaequalibus apice non caudato-acuminatis.

Arbor? ramis gracilibus teretibus superne leviter angulosis glabris; ramulis sulcatis obscure pubescentibus. Folia elliptica vel oblonga obtuse acuminata basi acuta $3-5 \mathrm{~cm}$. longa $1-3 \mathrm{~cm}$. lata initio membranacea denique coriacea, margine adpresse pubescentia mox 
glabrescentia; petiolis 7-10 $\mathrm{mm}$. longis rigidis supra canaliculatis pubescentibus. Flores $\uparrow$ racemoso-spicati, bracteis multifloris. Calyx 1-2 mm. altus, laciniis margine densius ciliato-pubescentibus dorso laxius puberulis; antheris crassis exertis $1 \mathrm{~mm}$. longis hirtellis. Flores $q$ : pedicellis $5-10 \mathrm{~mm}$. longis fructiferis $12 \mathrm{~mm}$. longis validiusculis ; calycis laciniis $11 / 2-2 \mathrm{~mm}$. longis mox deciduis oblongo-ellipticis apice rotundatis. Ovarium oblongo-cylindricum calycem 3-4-plo superantes sericeum. Drupa oblongo-ellipsoidea utrinque angustata $20 \mathrm{~mm}$. longa, $13 \mathrm{~mm}$. lata pilis densis albidis brevissimis tomentella.

Nom. JaP. Tsuge-modogi.

Type locality : Litkiu : insl. Okinawajima (lg. J. MatsuMURA! anno 1897, K. MiYake! anno 1899, Y. TASHIRo! anno 1897.) ; insl. Miyakojima (lg. S. TANAKA ! anno 1879.)

Range: Formosa? Ang-than-su? (leg. K. Miyake! Nov. 24, 1899.)

\section{Putranjixa integerrima Kordz. n. sp.}

Arbor 10-12 metralis 1-2 ped. crassus: ramis novellis glabris vetustioribus cinerascentibus. Folia glabra rigide coriacea supra vix nitidiuscula subtus opaca, elliptica apice rotundata rarius obtusissima, basi acuta raro late cuneata, integerrima, ad $6 \mathrm{~cm}$. lata et $71 / 2 \mathrm{~cm}$. longa; petiolis puberulis usque $10 \mathrm{~mm}$. longis mox glabris. Drupa ellipsoidea $19-25 \mathrm{~mm}$. longa 16-18 $\mathrm{mm}$. lata, luteo-velutina et pilis densis pallidis tomentella.

Nom. JAP. Hatsubaki.

Distr. Bonin : insl. Hahajima, Okimura (lg. S. Nishimura! no. 175 . Dec. 18,1918 ; lg. J. Toyoshima. no. 63.)

$\mathrm{Ab}$ omnibus speciebus foliis integerrimis distincta.

\section{Corydalis (Eucorydalis) brachystyla KoIDz. n. sp.}

Species habitu C. platycarpae MAK. sat similis, sed racemis plurifloris, capsulis brevioribus, stylis brevissimis dignoscenda.

Glaberrima flaccida circ. $30 \mathrm{~cm}$. alta, caule erecto e basi ascendente ramoso, ramis semper racemiferis. Folia tenuiter membranacea glaucoviridia, petiolis basi vaginato-dilatatis semiamplectentibus, inferioribus $81 / 2 \mathrm{~cm}$. longa, superioribus $3 \mathrm{~cm}$. longis; lamina bipinnatisecta $31 / 2-5$ cm. longis, $5-8 \mathrm{~cm}$. lata, segmentis primariis bijugatis erecto-patentibus, petiolis ad $13 \mathrm{~cm}$. longis, segmentis secundariis 1-2-jugatis sessilibus vel brevipetiolulatis, late ovatis trilobatis vel subtrilobatis; lobis ovatis mucronato-acutis basi cuneato-decurrentibus utrinque paucidentatis. Racemus pedunculo incluso 4-11 cm. longus ad 7-florus, floribus luteis 
$16 \mathrm{~mm}$. longis; pedicellis ad $9 \mathrm{~mm}$. longis, bracteolis lanceolatis acuminatis integris pedicello brevioribus. Sepala oblique cordato-ovata acuta pluri-dentata circ. 11/2-2 $\mathrm{mm}$. longa. Capsula cylindrico-subfusiformia $19 \mathrm{~mm}$. longa versus apicem leviter arcuata; stylis $1,2 \mathrm{~mm}$. longis, stigmatibus conspicuis, seminibus biseriatis nigris subreniformiorbicularibus compressis dense muricato-hispidis.

Nom. JAP. Shima-kikeman.

Distr. Bonin: insl. Chichijima (lg. J. Toyoshrma! no. 90, anno 1918.)

Euphorbia hirta Linn. Sp. Pl. (1753) p. 454;-Thunb. Fl. Jap. 196 ;-Merrill in Phil. Jour. Sc. IX. (1914) p. 101.

E. pilurifera LinN. ibid. 454 ;-Borss. in DC. Prodr. XV. 2, (1862) p. 21.

var. glaberrima Kordz. nov. var.

Planta glaberrima.

Distr. Bonin : Chichishima, Futagomura. (leg. S. Nishimura! no, 165, Aug. 23, 1918 ; no. 562. May 1915.)

Aconitum (Commaroidea) callianthum Kordz. nov. sp.

Species insignis nectariis erectis, casside prona rostro ascendente.

Humilis $26-36 \mathrm{~cm}$. alta manifeste flexuosa; foliis pedatim quinatis laciniis anguste lobatis, floribus pilosis, carpellis 3 glabris, filamentis ad medium bidenticulatis.

Tuber napiforme vel fusiforme fuscum $10-20 \mathrm{~mm}$. longum. Caulis $26-36 \mathrm{~cm}$. altus solitarius teres simplex glaber laevis superne flexuosus. Folia tenue coriacea utrinque parce puberula, pedatim quinata vel septenata, basi aperte cordata; lobis subrhombeo-ovatis acutissimis ternato-pinnatifidis; laciniis anguste linearibus vel lanceolato-linearibus acutis. Inflorescentia ad apices caulis subcorymbosa 3-7-flora, raro floribus ad axillas foliorum superiorum solitariis; floribus $3,5-4 \mathrm{~cm}$. longis intense violaceis; pedunculis erectis $2-3 \mathrm{~cm}$. longis robustis versus apicem subclavato-incrassatis, dense pubescentibus, bracteolis trifidis tenuiter pubescentibus lobis oblongis acutis. Fructus trini cylindrico-turgidi glabri $12-13 \mathrm{~mm}$. longi $5 \frac{1}{2} \mathrm{~mm}$. lati. Galea 22 $\mathrm{mm}$. longa vix compressa ad carinum et marginem dense peberula, dorso a basi sensim ampliata fere a medio abrupte et maxime prona, vertice rotundata, fronte profunde sinuata, rostro $8 \mathrm{~mm}$. longo, apice reflexo apertura maxime obliqua hiante. Sepala media $1 \frac{1}{1} 2 \mathrm{~cm}$. longa ac lata intus longe pilosa, sepala inferiora oblonga $14 \mathrm{~mm}$. longa apice rotundata intus tonge pilosula. Staminum filamenta lanceolata glabra 
subra medium bidenticulata, antheris rotundatis atro-violaceis. Carpella 3 glabra stylo ovario paulo breviore erecto apice obtuso. Nectaria valida glabra $2 \mathrm{~cm}$. longa, cuculli elongati erecti, calcare 5-6 $\mathrm{mm}$. longo abrupte reflexo apice revoluto, labio $5 \mathrm{~mm}$. longo versus apicem leviter dilatato binerve apice emarginato, tubo $5 \mathrm{~mm}$. longo.

Nom. Jap. Tsukushi-torikabuto.

Distr. Kiushiu: Prov. Hiuga, Nishiusuki-gōri, mt. Tōgatake (Iwadomura), leg. M. Ogata.

Wickstroemia pseudoretusa KoIDz. n. sp.

W. retusa Maxım. Mel. Biol. XII. 538 (quoad sp. ex Bonin!) non A. Gray.

Species sine dubio $W$. retusae valde affinis, sed ramis rugosis tomentosis breviter ramulosissimis, ramulis novellis fulvo-tomentosis, floribus minoribus pedunculis pedicellisque brevioribus, ovariis tomentosis longius stipitatis, stylis stigmatibusque minoribus.

Frutex dense ramosus, ramis nigris rugosis tomentosis vel mox glabrescentibus ramulis densis fulvo-tomentosis. Folia chartacea vel shartaceo-membranacea supra fere glaberrima subtus ad costas parce pilosa, obovalia obovata usque obovato-oblonga, 121/2-101/2, 21/2-1.7, 4.0-1.8 cm. longa et lata, integra, apice rotundata vel leviter retusa, basi late cuneata, nervis secundariis 7-circ. 10, supra planis subtus elevatis ; petiolis circ. $2 \mathrm{~mm}$. longis fulvo-tomentosis. Ca pitula pluriflora (5-7) ad apices ramulorum subsessilia, floribus luteis? circ. 9-10 $\mathrm{mm}$. longis, pedicellis, 1-1/2 mm. longis fulvo-tomentosis. Calyx carnosus extus dense pilosus tubo circ. $6 \mathrm{~mm}$. longo anguste cylindrico, lobis 4 ovatis vel elliptico-ovatis apice rotundatis interioribus $3 \mathrm{~mm}$. exterioribus $4 \mathrm{~mm}$. longis. Antherae 8 oblongae filamentis brevissimis; disci squamae 2 oblongae circ. $1,1 \mathrm{~mm}$. longae; ovaria stipitem fere aequantia. Pistillum $1.3 \mathrm{~mm}$. longum ovario tomentoso longe stipitato, stylis brevissimis stigmatibus capitatis. Fructus ovoideus circ. $7 \mathrm{~mm}$. longus basi pilosus.

Noм. JАP. Munin-awogampi,

Distr. Bonin: insl. Hahajima, Funanokiyama (lg. J.Toyoshima! no. 94.), insl. Chichishima, Shigureyama (lg. S. Nishimura! Oct. 1917.)

Claoxylon (Euclaoxylon) centenarium Kordz. n. sp.

Species perdistinctissima bracteis $\hat{\jmath}$ unifloris, staminibus circ. 110, petiolis elongatis.

Arbusculus? Folia alterna, petiolata, lanceolato-oblonga obtusa, basi oblique rotundata, margine repanda, glaberrima, in utraque parte costæ mediæ nervis secundariis circ. $10-11$ remotis erecto-patentibus 
ascendentibus deinde curvatis, intra marginem anastomosantibus, nervis utrinque fere planis, laxe reticulato-venosa, herbacea, supra viridia, subtus pallidiora, circ. $20-28 \mathrm{~cm}$. longa, $7-9 \frac{1}{2} \mathrm{~cm}$. lata; petiolis supra vix sulcatis glabris apice eglandulosis ultra $9 \mathrm{~cm}$. longis. Inflorescentia $\hat{\delta}$ axillares, solitariae, tenues, pedunculatae, racemiformes, laxe multiflorae, plus minusve puberulae, circ. $8-10 \mathrm{~cm}$. longae, pedunculo $1-2 \mathrm{~cm}$. longis, bracteis minutis dense puberulis. Flores $\hat{\delta}$ in axillis bracteae solitarii, pedicellati, pedicello dense puberulo, circ. 4-5 $\mathrm{mm}$. longo. Calyx profunde 3-partitus viridis, laciniis in praefloratione valvatis, inaequalibus, oblongis obtusis, concavis, intus glabris, extus dense puberulis, 4-5 mm. longis $2 \frac{1}{2}-3.0 \mathrm{~mm}$. latis. Stamina circ 110, toro leviter convexo inserta, in praefloratione erecta, tota $5 \mathrm{~mm}$. longa, antherae erectæ bipartitae extrorsae, thecis magnam partem liberis parallelis globoso-ellipsoideis longitudinaliter dehiscentibus, connectivo quam filamentum paulo crassiore; filamentis albis filiformibus tortuosis. Squamulæ lineares apice paucipilosae quam filamentum multo breviores. NoM. JAP.

Distr. Bonin : insl. Hahajima (leg. S. NishimURA! April. 1914.)

Pisonia Nishimurae Kordz. n. sp.

Arbor ramis tenuibus inermibus glaberrimis eglandulosis leviter striatis. Folia tenuiter membranacea glaberrima vix nitidula, linearioblonga 13-16 cm. longa, 6-7 cm. lata apice rotundato-emarginata basi cuneata, margine fere integerrima vel obscuriter repanda, nervis secundariis utrinsecus $8-10$ erecto-patentibus costa mediaque subtus leviter elevata, petiolis carnosis $21 / 2-3 \mathrm{~cm}$. longis glaberrimis. Inflorescentiæ terminales corymbosæ pedunculo communi $16 \mathrm{~cm}$. longo glaberrimo valido, ramis primariis 4 subumbellatis divaricatim patentibus glabris $3 \frac{1}{2}-5 \mathrm{~cm}$. longis apice umbellulas plurifloras pedunculatas subdivisis, basi foliis linearibus $4-6 \mathrm{~cm}$. longis $5-7 \mathrm{~mm}$. latis acutis basi longe attenuatis, pedicellis $3-5 \mathrm{~mm}$. longis perianthiisque extus dense fulvo-puberulis, bracteolis minutis squamosis, floribus $\hat{\delta}$. Perianthia e basi urceolata campanulatim ampliata ad $6 \mathrm{~mm}$. longa inferne $2 \mathrm{~mm}$. in ore ad $4 \mathrm{~mm}$. lata, limbis 5 subpatulis triangulariovatis obtusis. Stamina circ. 11 inæquilonga inclusa. Germen $5 \mathrm{~mm}$. longum ovario oblongo, stylo crassiusculo stigmate penicillato perianthium paullum sed distincte superante. Anthocarpia elongata vel globosa baccata facies sed imprimis circ. vertice minute et densius pulverulentihirtula, apice perianthii lobis erectis coronata.

NoM. JAP. Udoki.

Distr. Bonin, (leg. M. YaI! S. Niseimura!) 
Jasminum (Trifoliolata) superfluum Kordz. n. sp.

Frutex dense ramosus ramis fusco-brunneis pilis albis minutis recurvisque dense vestitis. Folia opposita trifoliolata, foliolis terminalibus ovato-oblongis 4-5 cm. longis, 1,8-2,5 cm. latis, 6-9 $\mathrm{mm}$. longe petiolulatis ; foliolis lateralibus oblique ovatis $1,5-3,0 \mathrm{~cm}$. longis, 9-16 mm. latis, brevissime (circ. $1 \mathrm{~mm}$. lg.) petiolulatis; omnibus apice obtusis usque obtusissimis mucronulatisque, basi obtusissimis usque rotundatis, integris, supra glabris subtus ad venas margineque minute puberulis ; nervis secundariis utraque latere $5-6$; petiolis $10-14 \mathrm{~mm}$. longis infra apicem subito geniculato-recurvis petiolulisque minute denseque puberulis. Flores graciles $3 \frac{1}{2} \mathrm{~cm}$. longi ad apices ramulorum dense cymosopaniculati, pedicellis $2-3 \mathrm{~mm}$. longis dense puberulis, bracteis bracteolisque squamosis fulvo-tomentosis. Calyx campanulatus $4 \mathrm{~mm}$. longus extus dense minuteque puberulus lobis 5 subulatis vel filiformibus $2 \mathrm{~mm}$. longis. Corolla hypocraterimorpha tubo elongato angusto 2,5 cm. longo $1 \frac{1}{2} \mathrm{~mm}$. lato, lobis 5 oblongo-linearibus acutis $10-11 \mathrm{~mm}$. longis $2 \frac{1}{2}-3 \mathrm{~mm}$. latis.

Nom. Jap.

Distr. Liukiu: insl. Okinawajima, Itomi (leg. T. Mryagr! no. 385.)

Salix rupifraga Kordz. n. sp.

Species S. hastatæ forsan affinis, sed bracteis apice haud crisposericeis, stylo pedicello stigmateque subæquilongis facillime dignoscenda.

Frutex rupestris ramis vetustioribus nigrescentibus rugosis valde verrucosis, ramulis brevibus badio vel luteo-brunneis cinereo-tomentosis raro glabris subnitidulis. Folia tenuiter coriacea juvenilia utrinque albo-villosa, adulta supra sordide viridia secus costas medias crispolanata, subtus pallida plus minus adpresse sericeo-pubescentia, ovatoelliptica vel ovato-oblonga rarius ovata vel lanceolato-oblonga acuta vel obtuse acutata, ad $6 \mathrm{~cm}$. longa et $2,5 \mathrm{~cm}$. lata, basi subrotundata usque obtusissima, margine glanduloso-denticulata; petiolis 3-4 $\mathrm{mm}$. longis albo-lanatis. Amenta fructifera ad $5 \mathrm{~cm}$. longa brevipedunculata basi foliis parvis ovatis vel obovato-oblongis obtusis subtus sericeovillosis 2-4 suffulta, cylindrica, densiflora, rhachi sericeo-pubescentia. Ovaria lanceolata glabra breviter stipitata, glandula una ventralis ovata obtusa stipitem aequilonga, bracteolis rotundatis albo-villosis stipitem æquilongis; stylis brevissimis, stigmatibus bipartitis laciniis vix bilobis.

NoM. JAP. Iwayanagi.

Distr. Nippon: Prov. Kai, Komagatake. 
Aster ageratoides Turcz. in Bull. Soc. Nat. Hist. Mosc. (1837.) VII. 154 ;-Walp. Rept. II. 574 ;-Maxim. Prim. Fl. Amur. suppl. Ind. Fl. Pekin. 472.

A. trinervius Auct. Plur. Jap. (non RoxB.)

var. alpinus Koidz. nov. var.

Monocephalus $16-24 \mathrm{~cm}$. altus erectus simplex dense foliatus; foliis sessilibus ovatis acutis $1-3 \frac{1}{2} \mathrm{~cm}$. longis utrinque circiter bidentatis, superioribus angustioribus, involucri phyllis latioribus obtusis glabrioribus, ligulis violaceis, pappo candido brevior robustior.

Nom. JAP. Takane-kongiku.

Distr. Nippon : Prov. Shinano, mt. Akashisan, alpibus.

\section{Angelica myriostachys KoIDz. n. sp.}

A. pubescenti valde affinis sed fructibus majoribus oblongis nec ovalirotundatis jam distinguenda.

Caulis elatus robustus ultra 4-pedalis striatus pubescens superne ramosus, ramulis erecto-patentibus floriferis umbellam fructiferam principalem superantibus. Folia ternato-bipinnatisecta, jugis 2-3, segmentis ultimis infimis.bi-tripartitis sectisve petiolulatis, terminalibus tripartitis anguste decurrentibus, omnibus membranaceis utrinque dense pubescentibus ellipticis vel oblongis raro ovatis, acutis serratis ; petiolis dense pubescentibus circ. $11 \mathrm{~cm}$. longis basi vaginato-amplexicaulibus, petiolis foliorum superiorum vaginato-ampliatis circ. $5 \mathrm{~cm}$. longis dense pubescentibus, involucro involucelloque nullis; umbellulis multifloris radiolis valde inaequalibus. Fructus oblongus $10-12 \mathrm{~mm}$. longus $5 \frac{1}{2}-61 / 2$ $\mathrm{cm}$. latus utrinque leviter emarginatus, jugis tribus dorsalibus nerviformibus, marginalibus alatis, valleculis dorsalibus $3-2$, lateralibus 3, commisularibus utrinque 3-vittatis, carpophoria bipartito. Antherac albae.

Nom. Jap. Tsukushi-kyokatsu.

HAB. Kiushiu : mt. Ubatake. 